\title{
Characterization of cell-cycle-specific events in synchronous cultures of Escherichia coli: a theoretical evaluation
}

\author{
N. B. Grover ${ }^{1}$ and Charles E. Helmstetter ${ }^{2}$ \\ Author for correspondence: N. B. Grover. Tel: +972 2758360. Fax: +972 2436890 \\ e-mail: norman@md2.huji.ac.il.
}

1 Hubert H. Humphrey
Center for Experimental
Medicine and Cancer
Research, The Hebrew
University Faculty of
Medicine, PO Box 12272,
Jerusalem 91120 , Israel
2 Department of Biological
Sciences, Florida Institute
of Technology, $150 \mathrm{~W}$
University Blvd,
Melbourne, FL 32901 , USA

\begin{abstract}
Synchronous growth studies are often used to assess the presence, timing and duration of periodic phenomena in the bacterial cell cycle. In an effort to evaluate the quality and quantity of information on cycle-specific events that can reasonably be expected from such inquiries, a model was constructed of a synchronous culture of Escherichia coli cells as would be derived from a growing population immobilized on a surface, and applied to the case of one stable and one unstable cellular component. The results indicated that, while the presence of cycle-specific events may be easily detectable, their timing and duration are very difficult to establish in synchronous growth experiments. Furthermore, differences in timing can be misconstrued as differences in duration, and vice versa, when interpretations are based on the qualitative analysis of the data.
\end{abstract}

Keywords: baby machine synchrony, cell-cycle-dependent gene expression, Escherichia coli division cycle, synchronous growth and cell division

\section{INTRODUCTION}

Studies on biosynthetic events in the Eschericbia coli cell cycle generally require the use of techniques to isolate or identify large numbers of cells in various stages of the division cycle. Early work in this area employed populations of cells that had been induced to grow and divide synchronously by a variety of means, such as temperature or nutritional shifts (Helmstetter, 1969). When it became evident that such harsh treatments disturbed normal biosynthetic processes, less stressful methods were devised to obtain synchronously growing cultures by selecting cells of relatively uniform age from exponentially growing populations. Two such techniques in current use involve selection of either the smaller cells or the newborn cells from a growing culture. The former can be isolated by centrifugal elutriation, a process in which a cell population in a centrifuge chamber is exposed to the opposing forces of sedimentation and fluid counter-flow. At the proper flow rate, the smaller cells are flushed from the chamber and will grow synchronously (Figdor et al., 1981). Newborn cells are provided by the 'baby machine', a device in which baby cells are released from a growing population immobilized on a surface (Helmstetter, 1969).

There are other ways to study molecular biosynthesis in the cell cycle that do not rely on synchronous growth at all but rather employ procedures for identification of the properties of cells of different ages in exponentially growing cultures. The baby-machine technique can be used for this purpose by labelling exponentially growing cells with precursors of the molecules of interest prior to immobilization and measuring the quantity of label in the newborn cells released continuously from the surface. By correlating these progeny cells with the age of their parents at the time of attachment, the properties of the molecules of interest can be determined as a function of cell age in the original, essentially undisturbed, culture. This approach was the primary element in the successful clarification of the relationships between DNA replication and the division cycle of $E$. coli (Helmstetter, 1967; Cooper \& Helmstetter, 1968). A second method involves flow cytometry, in which cell cycle properties are deduced from measurements on individual cells flowing, single file, past a focused light beam within a region of detectors (Edwards et al., 1992). This technique has proved particularly useful in investigating chromosome replication during the cell cycle (Allman et al., 1991). However, there are many studies in which the only currently feasible means of performing the work requires the use of synchronous cultures. In research on gene expression during the cell cycle, for instance, the rates of synthesis of unstable mRNAs cannot be measured effectively by either the backwards baby machine or flow cytometry, and such 
work is therefore routinely carried out on synchronous cultures (Sun \& Fuchs, 1992; Garrido et al., 1993; Theisen et al., 1993).

The purpose of the current inquiry was to analyse the quality and quantity of information on cell-cycle-specific events that can be expected from experiments on synchronous cultures of E. coli. The data were obtained from synchronous cultures generated with the baby machine, and consisted of measurements on the synthesis of one stable (plasmid DNA) and one unstable (a specific mRNA) component during the division cycle. Simulation studies, using parameters obtained from a least-squares fit to the synchronous growth data for newborn cells, were carried out to assess the level of attainable information on the timing of synthesis of these classes of macromolecules in the cell cycle.

\section{RESULTS AND DISCUSSION}

\section{The model}

The properties of synchronously dividing $E$. coli cells were modelled for the case of newborn bacteria collected from the effluent of a nitrocellulose-membrane-bound culture in a baby machine. The cell populations eluted from the membrane were taken as consisting of two components. The age of the major subpopulation was assumed to be normally distributed about zero with a standard deviation $\sigma$. The minor component, of relative size $\rho$, was considered to be a contaminant from a canonically distributed population on the membrane that had undergone only very little age selection during the elution process: the number of these random cells was set proportional to $2^{a / \tau}$, where $a$ is the age of the eluted cell at the time of collection $(t=0)$ and $\tau$ is the observed doubling time of the culture. These are bacteria released from the membrane for reasons other than division, such as weak binding. It also proved necessary to introduce a fixed background $\beta$ to account for the signals recorded by the electronic particle counter that were produced not by viable, replicating cells but by the debris and electronic noise that are invariably associated with such measurements.

This model, with the addition of a fourth adjustable parameter, the overall proportionality coefficient $k$, was fitted to the synchronous growth data for eluted cells of $E$. coli $\mathrm{B} / \mathrm{r}$ using an unconstrained nonlinear least-squares algorithm (IMSL, 1989). The results are presented in Table 1, and a plot of the experimental points together with the predicted curve is shown in Fig. 1(a). (Here and throughout, $\sigma$ is expressed as a percentage of $\tau$.)

The quality of the fit is rather good. The sum of squares of the residuals is $0.35 \%$ of the total $(P<0.001)$ and they are distributed randomly $(P>40 \%)$, as determined by the mean-square successive-differences test. The justification for employing a parametric test lies in the observation that the residuals are normally distributed $(P>20 \%$, as determined by the Lilliefors test); the weaker, nonparametric runs test gives a one-tail probability for randomness of $33.5 \%$.

\section{Synthesis of stable macromolecules during the division cycle}

The model was used to evaluate the analytical content in synchronous growth studies on the synthesis of stable macromolecules such as DNA, individual protein species, or specific stable RNAs. This was accomplished by comparing the predicted (best-fit) curve (Fig. 1a) to experimental data on the replication of plasmid DNA (Fig. 1b). The plasmid chosen for this purpose was the minichromosome pAL49, which harbours a copy of the chromosomal replication origin, oriC, as its sole origin of replication and has been shown to replicate at a specific

Table 1. Fit of model to experimental data

\begin{tabular}{|ccc|}
\hline Parameter & Value & Standard error \\
\hline$k$ & 0.989 & 0.030 \\
$\sigma$ & 0.146 & 0.012 \\
$\rho$ & 0.083 & 0.051 \\
$\beta$ & 0.136 & 0.022 \\
\hline
\end{tabular}

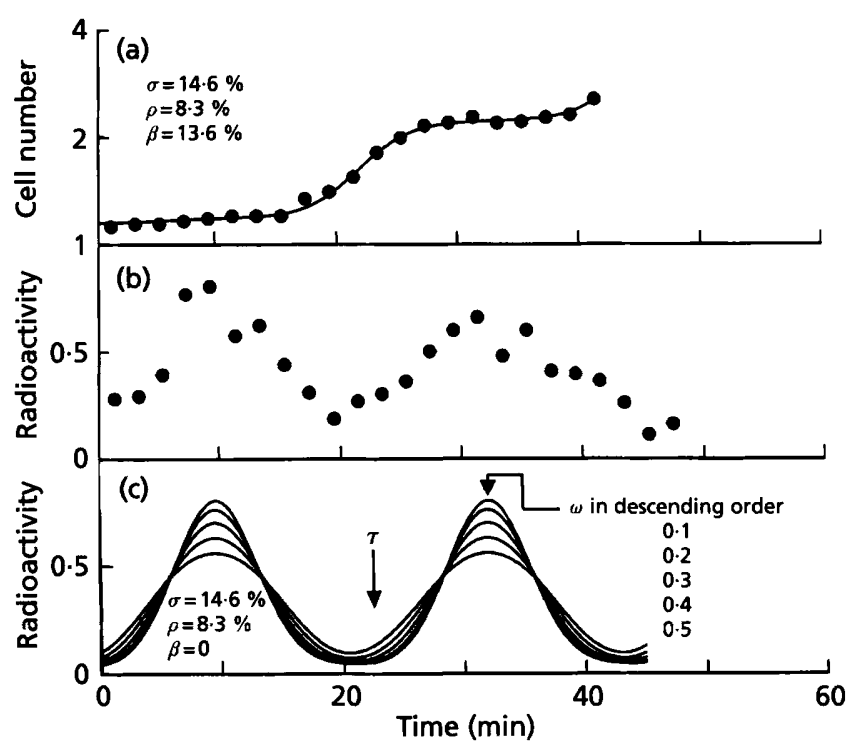

Fig. 1. (a) Number of $E$. coli $B / r$ cells eluted from a nitrocellulose membrane as a function of time modelled by combining three theoretical subpopulations: a major component with age normally distributed about zero (standard deviation $\sigma$ ), a contaminant that had undergone very little age selection (of relative size $\rho$ ), and a fixed background $(\beta)$ of noncellular material. Oxperimental results from Theisen et al. (1993), in which multiple, consecutive samples were collected from the effluent and grown for different times in order to generate synchronous growth data; - unconstrained nonlinear least-squares fit of model to data with parameter estimates as indicated. (b) Rate of pAL49 replication during synchronous growth of $E$. coli B/r, from Theisen et al. (1993). (c) Family of theoretical curves describing expected behaviour of stable macromolecular species, using parameter estimates for $\sigma$ and $\rho$ from (a) and $\beta=0$, for various values of the replication window $\omega$ as indicated. 


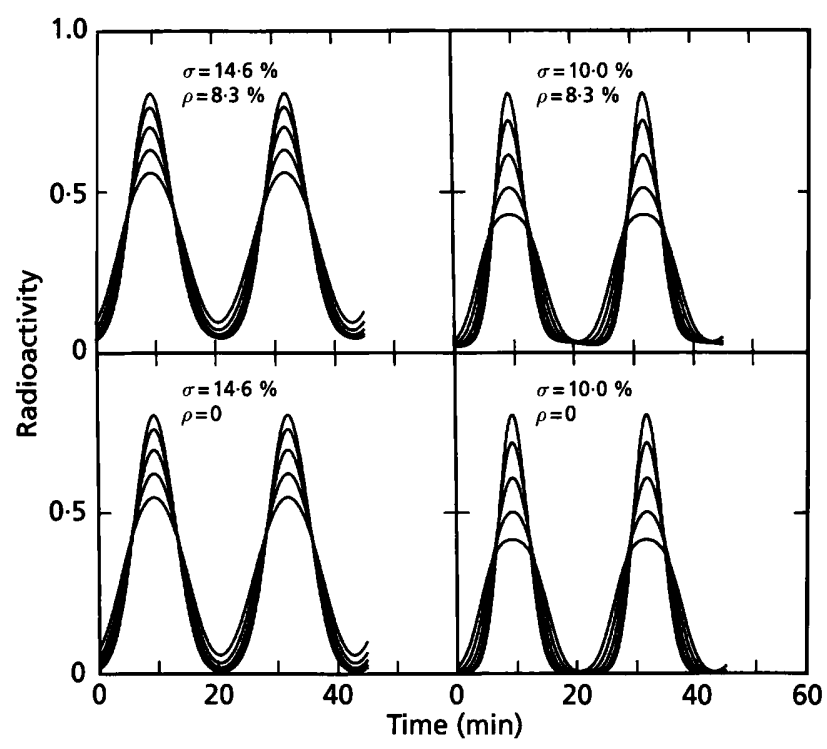

Fig. 2. Family of theoretical curves describing expected behaviour of stable macromolecular species, for various combinations of $\sigma$ and $\rho$ with $\beta=0$, using values of $\omega$ as in Fig. 1(c).

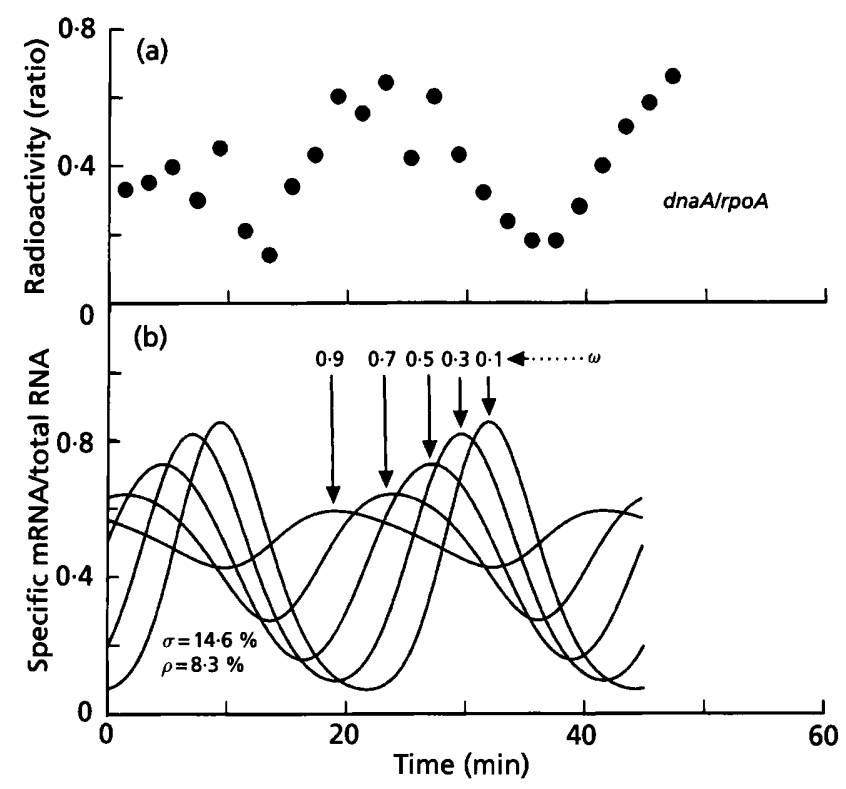

Fig. 3. (a) Level of $d n a A$ transcripts, relative to rpoA, during synchronous growth of $E$. coli $B / r$ (from Theisen et al., 1993). (b) Family of theoretical curves describing expected behaviour of unstable mRNA species, using parameter estimates for $\sigma$ and $\rho$ from Fig. 1(a) and $\beta=0$, for various values of the transcription window $\omega$.

time in the cell cycle, coincident with initiation of chromosome replication (Leonard \& Helmstetter, 1986). Replication of the minichromosome during synchronous growth was assayed by pulse-labelling cell samples with $\left[{ }^{3} \mathrm{H}\right]$ thymidine and measuring radioactivity incorporated into plasmid DNA by agarose gel electrophoresis and fluorography (Theisen et al., 1993). The minichromosome replicated periodically in the synchronous cultures, with the peaks of incorporation corresponding to the time of initiation of chromosome replication in the cycle. Fig. 1(c) contains a family of theoretical curves computed using the best-fit values of $\sigma$ and $\rho$ from Fig. 1(a) and a range of replication windows $\omega$ (which, like $\sigma$, is expressed in units of $\tau$ ) from $0 \cdot 1$ to $0 \cdot 5$.

Plasmid pAL49 is $7.6 \mathrm{~kb}$ in length and thus individual molecules would be expected to replicate in about $5 \mathrm{~s}$ if their rates of replication were the same as the average for the chromosome (Cooper \& Helmstetter, 1968). Since the labelling interval is approximately $10 \%$ of the mean doubling time, the experimental data can be expected to be comparable to the theoretical curve for a replication window of $0 \cdot 1$. The observed distribution is somewhat broader than the theoretical for $\omega=0 \cdot 1$ in the first cycle (and much broader in the second, but this is due to the decay of synchrony with time, which was not included in the model). It is evident that the experimental results could be considered consistent with any one of the predicted curves, and we conclude that it is not possible to determine the width of the window of synthesis for the minichromosomes by these means.

Fig. 2 illustrates the effects of different parameter values. The upper panels show the result of decreasing the dispersion in the synchronous population when the random component is as observed; the lower panels show the same but with the random component eliminated altogether (a feat rather easier to accomplish in silico than in vivo). The left-hand panels demonstrate the consequences of removing the random component when the dispersion of the synchronous population is as fit, and the right-hand panels demonstrate these consequences for the case of decreased dispersion. Even if such conditions were attainable, it is highly unlikely that the duration of the synthetic window could be estimated with any reasonable accuracy from comparable experimental data. It would certainly not be possible to do so simply by measuring the width of the distribution at the half-maximum of the peaks - the theoretical distributions are far too similar for so naive an approach.

\section{Synthesis of unstable macromolecules during the division cycle}

It has recently been shown that the expression of certain genes varies during the cell cycle. Transcription of $d n a A$ fluctuates due to an inhibition of transcription immediately after the gene replicates (Campbell \& Kleckner, 1990; Theisen et al., 1993). This inhibition is apparently caused by the binding of the newly-replicated, hemimethylated DNA to the cell membrane. Expression of the mioC gene is also periodic, again due to an inhibition of transcription. In this case, however, the inhibition was reported to begin shortly before replication of the gene, probably owing to the binding of DnaA protein in the promoter region. The timing of these periods of inhibition has been determined primarily in experiments in which 
initiation of chromosome replication was aligned by temperature shifts with $d n a C(\mathrm{ts})$ mutants. Periodicities in the expression of these two genes were also detected in baby-machine-generated synchrony experiments, and there too the peak in mioC transcription appeared to be slightly earlier than in $d n a A$ (Theisen et al., 1993).

The methodology described here was used to evaluate the information on the timing and duration of gene transcription in the cell cycle obtained from experiments with synchronously growing cultures of $E$. coli $B / r$. The results of one such experiment are presented in Fig. 3(a). The theoretical curves (Fig. 3b) were computed under the assumption that the rate of transcription is directly proportional to gene dosage. Based on the previous observations, initiation of replication was taken to occur $9.5 \mathrm{~min}$ into the division cycle, and mRNA half-life was set at $1.5 \mathrm{~min}$. The curves are drawn for various lengths of the period of inhibition of transcription from $0 \cdot 1 \tau$ to $0 \cdot 9 \tau$, all beginning at $9.5 \mathrm{~min}$. The data for $d n a A$ transcripts are similar to the theoretical curves for $\omega$ around $0 \cdot 7$, but greater precision is not possible. It is most remarkable, and highly unexpected, that the displacement of the experimental peak toward later times, as reported for dna $A$ with respect to mio $C$, could be explained by a longer duration of the inhibition, as well as by a difference in its timing as suggested previously (Theisen $e t$ al., 1993).

The primary rationale for this work was to ascertain whether synchronous cultures obtained with a wellcharacterized technique could be used effectively to determine the timing of events in the cell cycle, especially those that are difficult to measure, such as the pattern of gene expression. It is concluded that synchronous growth studies can be very useful for detecting the presence of periodicities during the cell cycle, but that the timing and duration of such events cannot be resolved by simple observation of the data alone. Even comparatively sophisticated analyses are not able to distinguish between an event in these experimental systems that occurs instantaneously in individual cells from one that occupies $20 \%$ or so of the cell cycle. Finally, the dispersion of even a truly instantaneous event must of necessity reflect the finite (and quite broad) age distribution of the eluted cells, and the recording of anything less should raise doubts as to the validity of the experimental assay in question.

\section{REFERENCES}

Allman, R., Schjerven, T. \& Boye, E. (1991). Cell cycle parameters of Eschericbia coli K-12. J Bacteriol 173, 7970-7974.

Campbell, J. \& Kleckner, N. (1990). E. coli ori $C$ and the dna $A$ gene promoter are sequestered from dam methyltransferase following passage of the chromosomal replication fork. Cell 62, 967-979.

Cooper, S. \& Helmstetter, C. E. (1968). Chromosome replication and the division cycle of Escherichia coli B/r. J Mol Biol 31, 519-540.

Edwards, C., Porter, J., Saunders, J. R., Diaper, J., Morgan, J. A. W. \& Pickup, R. W. (1992). Flow cytometry and microbiology. SGM Q Nov. 1992, 105-108.

Figdor, C. G., Olijhoek, A. J. M., Klencke, S., Nanninga, N. \& Bont, W. S. (1981). Isolation of small cells from an exponentially growing culture of Escherichia coli by centrifugal elutriation. FEMS Microbiol Lett 10, 349-352.

Garrido, T., Sanchez, M., Palacios, P., Aldea, M. \& Vicente, M. (1993). Transcription of $f t s Z$ oscillates during the cell cycle of Escherichia coli. EMBO J 12, 3957-3965.

Helmstetter, C. E. (1967). Rates of DNA synthesis during the division cycle of Escherichia coli B/r. J Mol Biol 24, 417-427.

Helmstetter, C. E. (1969). Methods for studying the microbial division cycle. Metbods Microbiol, 327-363.

IMSL (1989). Routine UNLSF, IMSL Math/Library, Version 1.1, pp. 835-840. Houston: IMSL.

Leonard, A. C. \& Helmstetter, C. E. (1986). Cell cycle-specific replication of Escherichia coli minichromosomes. Proc Natl Acad Sci US A 83, 5101-5105.

Sun, L. \& Fuchs, J. A. (1992). Escherichia coli ribonucleotide reductase expression is cell cycle regulated. Mol Biol Cell 3, 1095-1105.

Theisen, P. W., Grimwade, J. E., Leonard, A. C., Bogan, J. A. \& Helmstetter, C. E. (1993). Correlation of gene transcription with the time of initiation of chromosome replication in Escherichia coli. Mol Microbiol 10, 575-584.

Received 9 March 1994; revised 20 May 1994; accepted 24 August 1994. 